\title{
Protective effect of tulsi and levetiracetam on memory impairment induced by pregabalin on mice
}

\author{
Asher John Mohan, Krishna K. L.*, Jisham K. M., Seema Mehdi, \\ Ramesh B. Nidavani \\ Department of Pharmacology, JSS College of Pharmacy, JSS University, Sri Shivarathreeshwara Nagar, \\ Mysore -570015, Karnataka, India
}

\begin{abstract}
Memory impairment is the major concern of antiepileptic therapy. This study was undertaken to evaluate the memory impairment potential of Pregabalin (PGBL) and to assess the protective effect of hydro alcoholic extract of Ocimum sanctum leaves (OSHAE) and levetiracetam (LEV). Preliminary phytochemical screening and in-vitro antioxidant activity of OSHAE were done. Memory impairment induced by PGBL was evaluated by Morris water maze (MWM) test using mice as animal model. The animals were observed for Escape Latency Time (ELT) and Time Spent in Target Quadrant (TSTQ). OSHAE exhibited potent antioxidant and free radical scavenging activity. Chronic administration of $P G B L$ was found to induce memory impairment by increasing ELT and decreasing TSTQ when compared to normal animals. Memory impairment of PGBL was restored significantly by co-administration of OSHAE and LEV along with potentiation of anticonvulsant activity of PGBL. PGBL alone treated animals have shown more acetylcholinesterase (AChE) level when compared with normal animals where as co-administration of OS and LEV produced a decrease in AChE levels when compared to vehicle and PGBL treated group. The above study can be used as a reference to correct antiepileptic drug (AED) induced memory impairment by co-administration of nootropics without compromising on its antiepileptic potential.
\end{abstract}

Key-words: Levetiracetam, Memory impairment, Ocimum sanctum, Pregabalin

\section{Introduction}

Impaired memory is among the most common complaints of patients with epilepsy and multiple factors that contribute to memory impairment in patients with epilepsy [1]. The antiepileptic therapy enhances memory impairment in the patient and causes major set back. An antiepileptic therapy without or deceased memory impairment is much beneficial to the patient suffering from epilepsy. This can be achieved by co-administration of nootropic drugs but care has to be taken in retaining the antiepileptic potential. Pregabalin (PGBL) is a newer antiepileptic drug and the medical guide established by Pfizer which is approved by United States Food and Drug Administration (USFDA) classifies impaired memory/concentration as the most common side effect of PGBL. No scientific literature is available to report the memory impairment potential PGBL as on today. Ocimum sanctum (leaves) hydroalcoholic extract (OSHAE) has been proven as a memory enhancer [2] and an anti-convulsant [3]. Levetiracetam (LEV) a newer drug used to treat epilepsy [4] and has potential benefits for other psychiatric and neurological conditions such as Tourette syndrome, Alzheimer's disease (as a nootropic), autism, bipolar disorder and anxiety disorder [5].

Memory starts as a biochemical response in the brain following sensory input. Acetylcholine is a well known neurotransmitter, plays a critical synaptic role in the initial formation of memory. A transient change in neurotransmitter levels at synapses can be resulted in a short-term memory. It has been reported that levels of acetylcholine are deficient in the brains of patients with Alzheimer's disease, and what little acetylcholine produced is quickly broken down by the acetylcholinesterase (AChE) enzyme contributing to the loss of memory and other cognitive functions. Moreover, $\mathrm{AChE}$ is present in senile plaques in Alzheimer's disease brains. This parameter can be therefore employed in determining the degree of impaired memory of animals under study [6-7]. AChE levels in mice brain homogenate was performed in all treated animal groups to know the extent of memory deficit.

Keeping the above facts in view, the present study was conceived to evaluate the memory impairment activity of PGBL by chronic administration on mice, alone and in combination with anticonvulsant nootropics like OSHAE and LEV employing MWM test.

\subsection{Animals}

\section{Materials and Methods}

Albino mice of either sex (20-35 g) procured from the Central Animal House Facility of JSS Medical College, Mysore were used for the study. The animals were housed in polypropylene cages at $23-27^{\circ} \mathrm{C}$ with a natural light-dark cycle. The mice were fed on a standard mice pellet diet and water ad libitum. The animals 
were allowed to acclimatize to laboratory conditions for a week period before the start of the experiment. Groups of 6 mice were used in all sets of experiments. All the experiments were in accordance with the approval of Institutional Animal Ethics Committee (IAEC) of JSSCP Mysore; the project number is 123/2012.

\subsection{Drugs and chemicals}

All chemicals used for the study were procured from various suppliers and all were of analytical grade. PRBL and LEV were obtained as gift samples from Genvista Ahmedabad and Hetero Drugs, Hyderabad, India respectively. The OSHAE was procured from Sri Nidhi Industries, Mysore, Karnataka as gift samples.

\subsection{Preliminary phytochemical tests}

The preliminary phytochemical screening was carried out on OSHAE in order to find out the presence of various phytoconstituents [8-12].

\subsection{Antioxidant and free radical scavenging activity}

Antioxidant and free radical scavenging potential of OSHAE was evaluated by using in-vitro methodsSuperoxide anion radical scavenging assay [13], Hydroxyl radical scavenging assay [14], Nitric oxide radical scavenging assay (NO) [15], Ferric reducing antioxidant power assay (FRAP) [16] and DPPH radical scavenging assay [17].

\subsection{Memory impairment activity of PGBL on mice}

Evaluation of memory impairment activity of PGBL was done by using MWM test. The animals were divided into 5 groups of 6 animals each and treatments were given as shown in TABLE 1. The mice were released into the water and allowed for $60 \mathrm{sec}$ to find the platform. If the mouse does not find the platform after $60 \mathrm{~s}$, the animal was guided by putting finger on top of the platform and allowed to sit there for 10 seconds. Then the animal was placed in the heated cage for $60 \mathrm{sec}$ and afterwards next trial was started. Animals were returned to home cage after 4 trials. In general animals received three trials per day with five minutes inter-trial interval for seven days or until the performance was stable. Time to find the hidden platform was considered as Escape Latency Time (ELT). The platform in the water maze was kept at the same position throughout the test to assess the effect on spatial reference memory. TSTQ is defined as the time spent in the quadrant that previously contained the hidden platform was also measured [18].

\subsection{Anticonvulsant activity of PRBL}

The animals were treated as per the schedule (TABLE 1) for 29 days. Anticonvulsant potential of PGBL in absence/presence of OSHAE/LEV was assessed on $29^{\text {th }}$ day. Tonic and clonic convulsions were induced by giving MES ( $45 \mathrm{~mA}$ for $0.2 \mathrm{~s}$ ) using an electroconvulsiometer (Inco electroconvulsiometer) via ear electrodes. The nootropics as well as PGBL were given orally $2 \mathrm{hr}$ and $1 \mathrm{hr}$ respectively, before induction of MES. Duration of (a) flexion, (b) extension, (c) clonus, (d) stupor and or (e) recovery or death of animals was recorded [19].

\subsection{Estimation of brain AchE activity}

On $29^{\text {th }}$ day the animals were euthanized by cervical dislocation carefully to avoid any injuries to the brain tissue. The whole brain AChE activity was measured using the Ellman method [20]. The cloudy supernatant liquid $(0.5 \mathrm{ml})$ was pipetted out into $25 \mathrm{ml}$ volumetric flask and dilution was made with a freshly prepared dithiobisnitrobenzoic acid (DTNB) solution (10 mg DTNB in $100 \mathrm{ml}$ of phosphate buffer, $\mathrm{pH}$ 8.0). From the volumetric flask, two $4 \mathrm{ml}$ portions were pipetted out into two test tubes. Substrate solution $(1 \mathrm{ml})(75$ $\mathrm{mg}$ of acetylcholine iodide per $50 \mathrm{ml}$ of distilled water) was pipetted out into both tubes and incubated for 10 $\min$ at $30^{\circ} \mathrm{C}$.

The solution in the tube containing phosphate buffer was used for zeroing the colorimeter. The resulting yellow colour is due to reduction of DTNB by certain substances in the brain homogenate and due to non-enzymatic hydrolysis of substrate. After having calibrated the instrument, change in absorbance per minute of the sample was read at $420 \mathrm{~nm}$.

Table 1. Memory impairment activity of PRBL by Morris water maze model on MES induced convulsions. (Treatment schedule)

\begin{tabular}{|c|c|c|}
\hline Group & Treatment & Evaluation \\
\hline Normal+ MES & $\begin{array}{l}0.5 \%(2 \mathrm{ml} / \mathrm{kg}) \text { sodium CMC was administered } \\
\text { orally for } 29 \text { days and convulsions induced by } \\
\text { MES method. }\end{array}$ & $\begin{array}{l}\text { Durations of convulsion induced by MES method and } \\
\text { memory impairment activity were noted on } 8 \text { th, } 15 \text { th, } \\
\text { 22nd and } 29^{\text {th }} \text { day. }\end{array}$ \\
\hline PHT+MES & $\begin{array}{l}\text { PHT }(25 \mathrm{mg} / \mathrm{kg}) \text { was administered orally for } 29 \\
\text { days } 1 \mathrm{hr} \text { before convulsions induced by MES } \\
\text { method. }\end{array}$ & $\begin{array}{l}\text { Durations of convulsion induced by MES method and } \\
\text { memory impairment activity were noted on } 8 \text { th, } 15 \text { th, } \\
\text { 22nd and } 29^{\text {th }} \text { day. }\end{array}$ \\
\hline
\end{tabular}




\begin{tabular}{|c|c|c|}
\hline PRBL+ MES & $\begin{array}{l}\text { PRBL }(150 \mathrm{mg} / \mathrm{kg}) \text { was administered orally for } 29 \\
\text { days } 1 \mathrm{hr} \text { before convulsions induced by MES } \\
\text { method. }\end{array}$ & $\begin{array}{l}\text { Durations of convulsion induced by MES method and } \\
\text { memory impairment activity were noted on } 8 \text { th, } 15 \text { th, } \\
22 \text { nd and } 29^{\text {th }} \text { day. }\end{array}$ \\
\hline $\begin{array}{l}\text { PRBL+OSHAE+ } \\
\text { MES }\end{array}$ & $\begin{array}{l}\text { OSHAE }(200 \mathrm{mg} / \mathrm{kg}) \text { and PRBL }(150 \mathrm{mg} \mathrm{kg}-1) \\
\text { was administered orally for } 29 \text { days } 2 \mathrm{hr} \& 1 \mathrm{hr} \\
\text { before convulsions induced by MES method. }\end{array}$ & $\begin{array}{l}\text { Durations of convulsion induced by MES method and } \\
\text { memory impairment activity were noted on } 8 \text { th, } 15 \text { th, } \\
22 \text { nd and } 29^{\text {th }} \text { day. }\end{array}$ \\
\hline $\begin{array}{l}\text { PRBL+ } \\
\text { MES }\end{array}$ & $\begin{array}{l}\mathrm{LEV}(400 \mathrm{mg} / \mathrm{kg}) \text { and PRBL }(150 \mathrm{mg} / \mathrm{kg}) \text { was } \\
\text { administered orally for } 29 \text { days } 2 \mathrm{hr} \& 1 \mathrm{hr} \text { before } \\
\text { convulsions induced by MES method. }\end{array}$ & $\begin{array}{l}\text { Durations of convulsion induced by MES method and } \\
\text { memory impairment activity were noted on } 8 \text { th, } 15 \text { th, } \\
22 \text { nd and } 29^{\text {th }} \text { day. }\end{array}$ \\
\hline
\end{tabular}

Note- All group animals were subjected for MES induced convulsion on $8^{\text {th }}, 15^{\text {th }}, 22^{\text {nd }}$ and $29^{\text {th }}$ day of study

\subsection{Preliminary phytochemical screening of OSHAE}

\section{Results}

Phytochemical screening of OSHAE indicated presence of alkaloids, triterpenoids, tannins, flavonoids, cardiac glycosides and trace amounts of carbohydrates.

\subsection{In-vitro antioxidant activity of OSHAE}

In-vitro antioxidant and free radical scavenging activity revealed that, OSHAE was found to have good antioxidant and potent scavenger of superoxide, hydroxyl, nitric oxide and DPPH radicals. Additionally it exhibited good ferric reducing power when evaluated by ferric reducing antioxidant power assay (TABLE 2).

\subsection{Memory impairment activity of PRBL}

The memory impairment induced by chronic administration of PGBL and protective effect of OSHAE and LEV is depicted in Fig. 1, $2 \& 3$. The activity was done by MWM test using mice as experimental animal and ELT, TSTQ and whole brain acetyl cholinesterase activity were the parameters evaluated.

Table 2. Antioxidant activity of OSHAE by in-vitro methods

\begin{tabular}{lll}
\hline \multirow{2}{*}{ Method } & IC $_{\mathbf{5 0}}$ Value $(\square \mathbf{g} / \mathbf{m l})$ \\
& OSHAE & Ascorbic acid \\
\hline Hydroxyl radical scavenging assay & $210.08 \pm 0.60$ & $4.83 \pm 0.04$ \\
Nitric oxide radical scavenging assay & $195.19 \pm 0.33$ & $5.24 \pm 0.15$ \\
Ferric reducing antioxidant power assay & $112.53 \pm 0.20$ & $4.15 \pm 0.15$ \\
Superoxide anion radical scavenging assay & $416.67 \pm 0.12$ & $5.66 \pm 0.25$ \\
\hline
\end{tabular}

\section{Values are Mean \pm SD of triplicate}

It was observed that when PRBL was administered orally for 29 days on mice with MES induced convulsions, there was a significant increase in ELT $(86.21 \pm 1.26 \mathrm{~s})$ when compared to the normal group $(9.65 \pm 2.50 \mathrm{~s})$. Memory impairment of PRBL was found to be almost similar to PHT (an antiepileptic drug that has been proven to produce memory impairment in animal models). The study reveals that co-administration of OSHAE with PRBL on MES induced convulsions resulted in a significant decrease in ELT to $8.15 \pm 2.25 \mathrm{~s}$ when compared to the PRBL, PHT as well as normal treated group. It was observed that co-administration of LEV at dose of $400 \mathrm{mg} / \mathrm{kg}$ along with PRBL with MES induced convulsions resulted in a significant decrease in ELT of $7.12 \pm 1.33 \mathrm{~s}$ when compared to the PRBL, PHT and normal group animals. The results obtained clearly proves that OSHAE and LEV have improvement in learning and memory in mice brain and reversed the PRBL induced memory impairment with LEV having an upper hand in nootropic activity (Fig. 1).

TSTQ is another parameter recorded for evaluating memory deficit property of PRBL and protective effect by co-administration of OSHAE and LEV. It was observed that chronic administration of PRBL in mice for 29 days with MES induced convulsions resulted in significant decrease in TSTQ to $5.24 \pm 1.04 \mathrm{~s}$ when compared to the normal group (49.15 $\pm 3.18 \mathrm{~s})$. Extent of memory impairment was found to be similar among PRBL and PHT. Co-administration of OSHAE extract at the dose of $200 \mathrm{mg} / \mathrm{kg}$ with PRBL on MES induced convulsions resulted in a significant increase in TSTQ of $49.35 \pm 2.03 \mathrm{~s}$ when compared to the PRBL alone treated animals. This shows that, the OSHAE has potent memory improving activity on PRBL induced memory deficit. Co-administration of LEV at the dose of $400 \mathrm{mg} / \mathrm{kg}$ with PRBL on MES induced convulsions resulted in a significant increase TSTQ 55.69 \pm 2.18 seconds when compared to the PRBL treated animals. The results hence prove the ability of OSHAE and LEV in reducing the memory deficit produced by anticonvulsant drug PRBL (Fig. 2). 


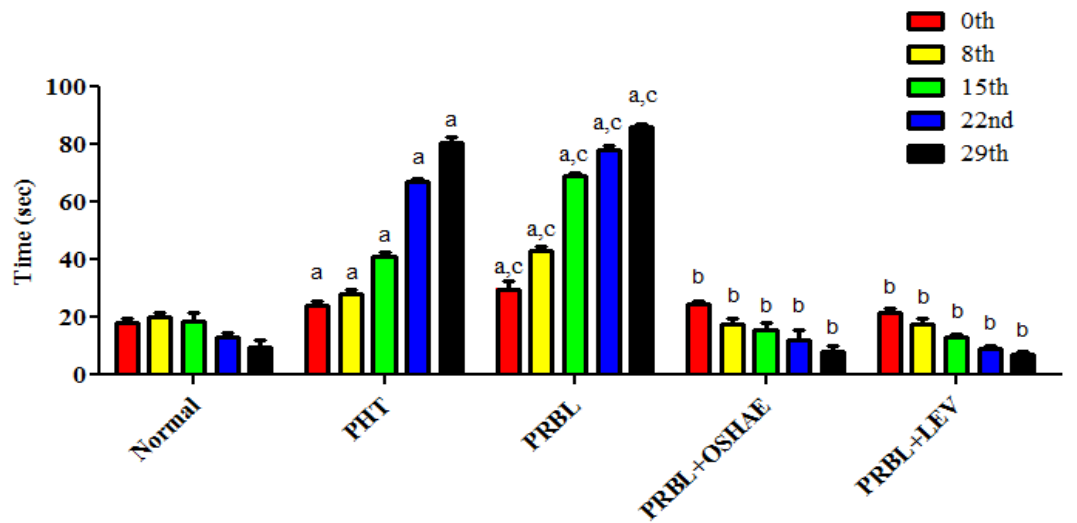

Values are expressed as Mean \pm SEM, $n=6$ $a$ - Significant when compared with normal animals $(P<0.05)$

$b$ - Significant when compared with PRBL group animals $(P<0.05)$

$c$ - Significant when compared to PHT group animals $(P<0.05)$

Figure.1- Memory impairment activity of PRBL and protective effect of OSHAE and LEV (Escape Latency Time in seconds-ELT)

\subsection{Estimation of brain AChE levels}

It was observed that administration of PRBL along with MES induced convulsions resulted in a significant $(P<0.05)$ increase in AChE when compared to the normal group. OSHAE and LEV coadministration with PRBL in MES induced animals, significantly decreased AChE concentration compared to PRBL alone treated animals. This indicates that, there is low level of acetylcholine in PRBL group animals, which was restored by co-administration of OSHAE and LEV (Fig. 3).

\subsection{Anticonvulsant activity}

Flexion, extension, clonus, stupor, death or recoveries were the parameters used to evaluate anticonvulsant activity of PRBL on MES induced convulsion. When PRBL was administered orally for 29 days, it significantly produced protection in extension phase $2.07 \pm 1.09 \mathrm{~s}$ and decreased the duration of stupor to $32.20 \pm 1.16 \mathrm{~s}$ when compared to normal group animals. It was observed that when OSHAE was co-administered with PRBL resulted in $100 \%$ protection in extension phase $(0.00 \pm 0.00 \mathrm{~s})$ and decreased duration of stupor to $12.44 \pm 1.18 \mathrm{~s}$ when compared to normal group thus indicating synergistic phenomenon. On the other hand LEV when administered along with PRBL did not produce any synergistic effect in extensor phase but reduced the duration of stupor which was found to be similar to PRBL alone treated animal group. The result clearly shows that PRBL retained its protection on MES induced convulsion even when co-administered with OSHAE and LEV.

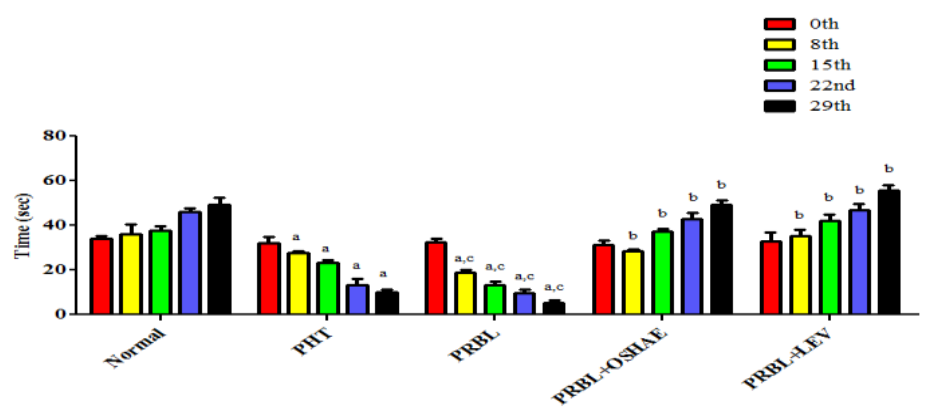

Values are Mean \pm SEM, $n=6$,

$a$ - Significant when compared with normal animals $(P<0.05)$

$b$ - Significant when compared with PRBL group animals $(P<0.05)$

$c$ - Significant when compared to PHT animals $(P<0.05)$

Figure.2- Memory impairment activity of PRBL and protective effect of OSHAE and LEV (Time Spent in Target Quadrant in seconds-TSTQ) 


\section{Discussion}

Epilepsy is chronic neurological disorders characterized by seizures which results from abnormal, excessive or hyper synchronous neuronal activity in the brain [21]. Onset of new cases occurs most frequently in infants and the elderly. PRBL is one of the newer AED used as an adjunct therapy for partial seizures with or without secondary generalization causing memory impairment [22].

Many herbs have both nootropic as well as anticonvulsant activity which includes Withania somnifera, Ocimum sanctum Linn [23] etc. which can be used for correcting the memory deficit caused by anticonvulsant drugs as well as potentiate the anticonvulsant activity. As stated in the earlier section, the present study was conceived to evaluate the memory deficit property of PRBL and to assess the protective effect of OSHAE and LEV.

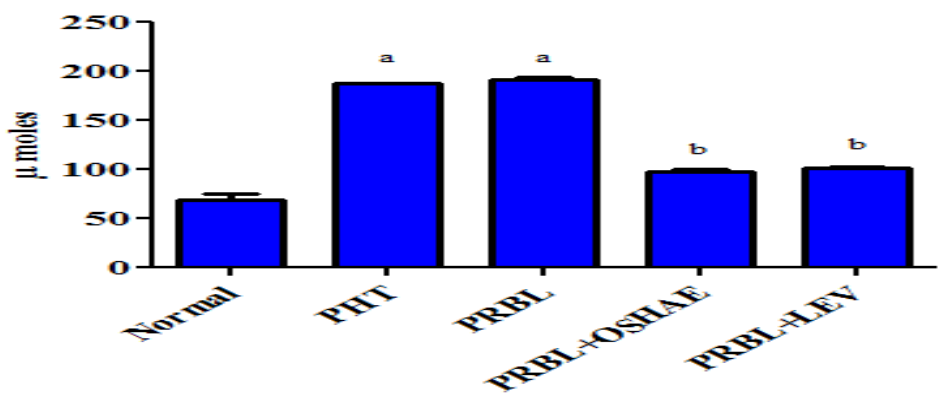

Values are Mean \pm SEM, $n=6$,

$a$ - Significant when compared with Normal animals $(P<0.05)$

$b$ - Significant when compared with $P R B L$ group animals $(P<0.05)$

Figure.3- Memory impairment activity of PRBL and protective effect of OSHAE and LEV (brain AChE levels)

In order to assess the memory impairment potential of PRBL and protective effect of OSHAE and LEV we used MWM test. The essential feature of the test employed is that mice are placed into a large circular pool of water and can escape onto a hidden platform. The platform is hidden by its placement just below the water surface and by opaque water. Thus the platform offers no local cues to guide the escape behavior. The mice can escape from swimming by climbing onto the platform and over time the mouse apparently learns the spatial location of the platform from any starting position at the circumference of the pool. The only spatial cues are those outside of the water tank and are primarily visual cues. MWM performance involves several components, including concept formation (learning the general rules of the task), attention, working memory and reference memory, which are not readily distinguishable in a simple form of this paradigm. MWM offers several advantages over other models of learning and memory such as absence of motivational stimuli such as food and water deprivation, electrical stimulations and buzzer sounds (as required in passive avoidance task), which may potentially affect memory evaluation test [24].

The results of the present study showed that PRBL when administered orally for 29 days along with MES induced convulsions, significantly increased the ELT by $144.70 \%, 231.31 \%, 261.20 \%, 287.84 \%$ and decreased TSTQ by $16.95 \%, 29.25 \%, 40.58 \%$ and $57.98 \%$, on $8^{\text {th }}, 15^{\text {th }}, 22^{\text {nd }}$ and $29^{\text {th }}$ day of treatment respectively when compared to normal group animals. The result clearly demonstrates that, the PRBL adversely affected cognitive impairment in the MWM task in mice (Fig. $1 \& 2$ ). These findings show that, the PRBL treatment in mice induces memory impairing effects.

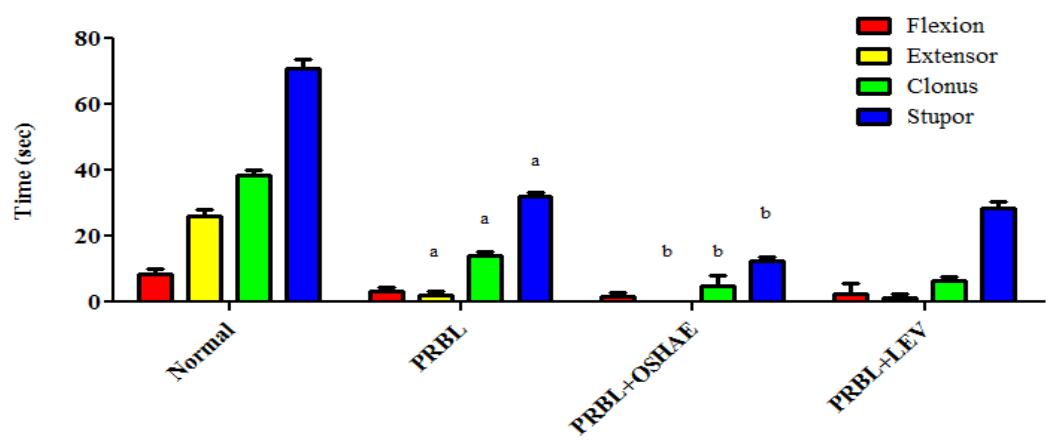

Values are Mean \pm SEM, $n=6$,

$a$ - Significant when compared with Normal treated animals $(P<0.05)$

$b$ - Significant when compared with PRBL treated animals $(P<0.05)$

Figure.4- Anticonvulsant activity of PRBL in presence and absence of nootropics on mice by MES induced convulsions $\left(29^{\text {th }}\right.$ day of treatment) 
Our study supports the available literature regarding the memory impairment activity of PRBL [25]. The protective effect produced by OSHAE may be due to its strong antioxidant and free radical scavenging potential (TABLE 2).

The memory impairment produced by PRBL is compared with PHT treated group which has already been proven for its memory deficit activity [26]. By all parameters PRBL produces same extent of memory impairment as that of PHT.

The whole brain AChE activity was measured on the basis of the formation of yellow colour due to the reaction of thiocholine with dithio-bis-nitrobenzoate ions. The rate of formation of thiocholine from acetylcholine iodide in the presence of tissue cholinesterase was measured using a spectrophotometer. In the current study we found that, the mice treated alone with PRBL have shown more amount of AChE enzyme whereas the co-administration with OSHAE and LEV restored the elevated level of AChE to normal (Fig. 3). This reveals the protective effect of OSHAE and LEV on impaired memory induced by chronic administration of PRBL on mice. The nootropic effect of OS maybe attributed to its antioxidant property. While the actual mechanism by which LEV may act as a nootropic isn't known, a few assumptions are by either reducing the abnormal spike activity or reversing hippocampal remodeling, behavioral abnormalities, synaptic dysfunction, and deficits in learning and memory [27].

MES induced convulsions in animal represent grand mal type of epilepsy. In MES convulsions, electroshock was applied through the ear electrodes. Through cochlear nerve stimulation, cortical excitation was produced. Drug which reduces or totally abolishes the extension phase is considered to be antiepileptic drug [19].

In the present study we have evaluated the anticonvulsant activity of PRBL in the presence and absence of nootropic herb OSHAE and LEV (Fig. 4). Some recent studies report anticonvulsant effects of OSHAE against MES induced seizures [28-29]. Here it was observed that PRBL retained its antiepileptic activity when co-administered with LEV while its activity was potentiated with OSHAE. This study clearly demonstrates the protective effect as well as synergistic effect of OS on PRBL for grand mal type of epilepsy on which PRBL alone show less significant antiepileptic activity. However further studies are required to strongly establish the hypothesis involved in the current findings

\section{Conclusion}

The study was thereby concluded that chronic administration of PRBL was found to induce memory impairment in mice when tested on MWM test. The memory impairment produced by PRBL was found to be as that of PHT a standard antiepileptic drug which was very well established for its memory impairment adverse effect. OSHAE \& LEV can be employed as antiepileptic nootropics to correct the memory deficit induced by PRBL without compromising on its antiepileptic activity. Even co-administration of OSHAE has synergized the anti-epileptic activity of PRBL on MES induced epileptic mice. Further clinical investigation can bring new memory impairment free line of therapy for treatment of epilepsy.

\section{Acknowledgement}

The authors sincerely thank Dr. H.G. Shivakumar, Principal, JSS College of Pharmacy, Mysore, for his support and encouragement. Our gratitude goes to JSS University, Mysore, for providing all the necessary facilities. The authors also thanks, Sri Nidhi Industries, Mysore for supply of tulsi extract.

\section{References}

[1] A. C. Joyce, M. Scott, W. James, and H.M. Richard Adverse effects of antiepileptic drugs: a brief overview of important issues, Expert Rev Neurother, 10(6), 2010, 885-891.

[2] H. Joshi, and M. Parle, Cholinergic basis of memory improving effect of Ocimum tenuiflorum Linn, Indian J Pharm Sci, 68(3), 2006, 364-365.

[3] R.K. Jaggi, R. Madaan, and B.Singh, Anticonvulsant potential of holy basil, Ocimum sanctum Linn. and its cultures, Indian J Exp Biol, 41(11), 2003, 1329-1333.

[4] B. Abou Khalil, Levetiracetam in the treatment of epilepsy, Neuropsychiatry Disease Treatment, 4(3), 2008, 507-523.

[5] Internet search- Accessed on December 15 ${ }^{\text {th }}, 2012-$ http://www.pnas.org/content/suppl/2012/08/06/1121081109. DCS upplemental

[6] A.C. Marcelo, E.R. Ariel, and N.C. Inestrosa, Acetylcholinesterase induces neuronal cell loss, astrocyte hypertrophy and behavioural deficits in mammalian hippocampus. J Neurochem, 87, 2003, 195-204

[7] Internet search- Accessed on December $15^{\text {th }}, 2012$ http://intelegen.com/nutrients/memory_enhancement_and_cognitive.htm

[8] C.K. Kokate, A.P Purohit, and S.B. Gokhale, Pharmacognosy (Nirali Prakashan: Pune.1996).

[9] I.L. Finar, Organic chemistry (Longman Scientific and Technical, London: 1975)

[10] G.E. Trease, and W.C. Evans, Pharmacognosy (Elsevier Health Sciences, London: 1989).

[11] T.A. Geinssman, Flavanoids, In Modern Methods of Plant Analysis (Berlin: Springer Verlag: 1955).

[12] B.T. Cromwell, Alkaloids. In Modern Methods of Plant Analysis (Berlin: Springer Verlag: 1955).

[13] W.S. Chang, C.C. Lin, S.C. Chuang, and H.C. Chiang, Superoxide anion scavenging effect of coumarins, American Journal of Chinese Medicine. 24, 1996, 11-17. 
[14] E. Kunchandy, and M.N. Rao, Oxygen radical scavenging activity of curcumin. International Journal of Pharmaceutics, 58, 1990, $237-240$.

[15] L.C. Green, D.A. Wagner, J. Glogowski, P.L. Skipper, J.S. Wishnok, and S.R. Tannenbaum, Analysis of nitrate, nitrite and 15N nitrate in biological fluids, Analytical Biochemistry, 126, 1982, 131-138.

[16] I.F. Benzie, and J.J. Strain, The ferric reducing ability of plasma (FRAP) as a measure of "antioxidant power" the FRAP assay, Analytical Biochemistry, 239, 1996, 70-76

[17] P. Mandal, T. Kumar, and M. Ghosal Free-radical scavenging activity and phytochemical analysis in the leaf and stem of Drymaria diandra Blume, International Journal of Integrative Biology, 7(2), 2009, 80-84.

[18] G. Achliya, U. Barabde, S. Wadodkar, and A. Dorle, Effect of Brahmi Ghrita, a polyherbal formulation on learning and memory paradigms in experimental animals, Indian J Pharmacol 36(3), 2004, 159-162.

[19] S.K.Kulkarni, Practical Pharmacology and Clinical Pharmacy, (Vallabh Publications, Delhi: 2005).

[20] G.L. Ellman, K.D. Courtney, A. Valentino, and R.M. Featherstone, A new and rapid colorimetric determination of Acetylcholinesterase activity, Biochem Pharm, 7, 1961, 88-95.

[21] M.J. Brodie, A.T. Elder, and P. Kwan, Epilepsy in later life, Lancet, 8(11), 2009, 1019-1030.

[22] Internet search- Accessed on January $27^{\text {th }} 2013, \quad$ http://www.ehealthme.com/ds/pregabalin/memory+impairment

[23] K.D. Subir, and D.M. Vasudevan, Tulsi: The Indian holy power plant. Natural Product Radiance, 5(4), $2006,279-283$.

[24] M.K. Saraf, and S. Prabhakar Anand. Bacopa monniera alleviates N-(omega)-nitro-L-arginine induced but not MK-801-induced amnesia: a mouse Morris water maze study. Neuroscience 2009; 160(1): 149-55.

[25] Internet search- Accessed on October $29^{\text {th }}, 2012$ -

http://www.drugs.com/sfx/Pregabalin-side-effects.html

[26] D. Vohora, S.N. Pal, and K.K. Pillai, Protection from phenytoin-induced cognitive deficit by Bacopa monniera, a reputed Indian nootropic plant, J Ethnopharmacol, 71(3), 2000, 383-390.

[27] E. Pascal, Z. Lei et al. Levetiracetam suppresses neuronal network dysfunction and reverses synaptic and cognitive deficits in an Alzheimer's disease model. Proc Natl Acad Sci, 2012; 109(42):E2895-2903.

[28] J.B. Shraddha, Krishna KL. Protective effect of Nootropics on Memory impairment induced by an anti-epileptic., Master diss., JSS University, Mysore, 2012.

[29] J. Sethi, Sushma S, Shashi S, Anjana T. Evaluation of hypoglycemic and antioxidant effect of Ocimum sanctum. Indian Journal of Clinical Biochemistry 2004; 19(2): 152-155. 